\title{
HxI : An Australian Initiative in ICT-Augmented Human Interactivity
}

\author{
Christian Müller-Tomfelde ${ }^{1,2}$, Belinda $\operatorname{Kellar}^{1}$, and Peter Eades ${ }^{1}$ \\ ${ }^{1} \mathrm{HxI}$ Initiative - [braccetto] Project \\ ${ }^{2}$ CSIRO ICT Centre \\ \{firstname. lastname\}@hxi.org.au
}

\begin{abstract}
The nature of global business today means people often need to work as part of geographically dispersed teams. As such, organisations around the globe are looking to improve the way employees collaborate and share knowledge, even if they are collaborating across large distances. The complexity of dealing with distributed knowledge workers is heightened by the increasing struggle by individuals to extract and make sense of the huge amounts of data that needs to be processed in order to extract meaningful insights within their work context. The HxI Initiative is a new national initiative in Australia, which is driven by these overarching business drivers. Research is planned and conducted to improve the ability of humans to interact with information, their colleagues and their environments in the modern organization.
\end{abstract}

\section{Overview}

The HxI Intiative was established in 2006 by three of Australia's publicly funded research organisations - Commonwealth Scientific Research Organisation (CSIRO), Defence Science Technology Organisation (DSTO) and National ICT Australia (NICTA) as a new national initiative in ICT-Augmented Human Interactivity. The initiative is distributed across Australia, with key sites in Sydney and Adelaide (see also [1]).

The term 'HxI' describes the trend towards ubiquity and human experience in information communications technology (ICT) environments. Specifically, the " $x$ " in the term 'HxI' represents research from a number of disciplines that collectively enhance "the factor of human interactivity". In this way, the HxI Initiative's research is focused on augmenting the ability of humans to interact with information, with each other, and with their environments through the effective application of ICT [3]. This includes research into the development of innovative interaction technologies; novel interactive visualisation approaches, ubiquitous computing environments, and integrated telepresence for cooperative work.

The aims of the HxI Initiative include not only the development of world-class scientific and industrial outcomes in the field, but at a strategic level the development of human capital in this multi-disciplinary field. This includes developing strategies to build Australia's presence in the field of human interactivity; reducing fragmentation 
across related research disciplines and building human capital through targeted recruitment and research training in the HxI field.

\section{Foundational Project [braccetto]}

The [braccetto] project is the first and foundational project of the HxI Initiative. The project is the largest HCI related project ever undertaken in Australia. It began in late 2006.

The [braccetto] project is exploring how the effective application of ICT in mixed presence groupware can help geographically distributed teams collaborate more effectively [2]. Specifically, the project is developing new methods for supporting simultaneous work between multiple people at multiple sites, using software tools tightly coupled to high-quality, multi-party telepresence technology. The project will design and evaluate tools and models of distributed teamwork. The eventual aim is to improve criteria such as productivity, performance and team effectiveness between remote co-workers.

The project is investigating a range of novel mixed presence groupware capabilities, including the development of a research and transitioning platform called [braccetto] TeamNets. [braccetto] TeamNets is a family of hardware and software elements that can be rapidly composed into a range of distributed collaboration systems. It includes the use of high quality audio and video communication, synchronous application sharing over a distance and rapidly configurable, adaptable and synchronised operating environments $[2,3]$. A real-world domain experiment in the national security field, was undertaken in November 2006 and results from this study and underlying research into awareness and mixed-presence groupware is providing stimuli to the development of new models and systems for distributed intense collaboration environments. Further large scale experiments are planned.

Future research to be addressed by the [braccetto] project includes: combining remote awareness cues (communicative gestures, direction of attention) with a generic groupware platform for arbitrary applications; conducting large-scale formative evaluations of a combined high quality telepresence, groupware and ubiquitous computing system; and assessing situation awareness and workspace awareness in mixed presence groupware applications for geographically dispersed teams.

\section{References}

1. HxI Initiative (accessed 24, February 2007) (2007), http://www.hxi.org.au/

2. Schremmer, C., Krumm-Heller, A., Vernik, R., Epps, J.: Design Discussion of the [braccetto] Research Platform: Supporting Distributed Intensely Collaborating Creative Teams of Teams. In: Proc. HCI International 2007 (2007)

3. Vernik, R., Kellar, B., Epps, J., Schremmer, C.: HxI: A National Research Initiative in ICTAugmented Human Interactivity. Internal Report (accessed April 18, 2007) (2006), http:/www.hxi.org.au/images/stories/documents/verniket_al_oct06.pdf 